\title{
Participatory Action Research Approaches and Methods
}

Sara Kindon, Rachel Pain \& Mike Kesby (eds), Routledge, New York \& London, 2007, 288 pages

\author{
Nancy Gibson \\ University of Alberta
}

Gateways: International Journal of Community Research and Engagement Vol 3 (2010): 185-186 (C)TSePress and the author

ISSN 1836-3393
This book, published as part of Routledge's Studies in Human Geography, is useful well beyond this discipline, as it provides a welcome review of Participatory Action Research (PAR). In three major sections, beginning and ending with 'Reflections' that bracket the 'Action' section, this edited collection provides a timely overview of the current status of this methodology, as well as many useful examples of applying PAR as a research process. The papers build on the work of Cooke and Kothari (2001) and others who forced us to take a fresh look at the PAR approach. The collection addresses the ideological concept behind participatory research and adds reflection and experience to outline a tangible method that can be described and evaluated, and consistently applied. The book documents the shift from a focus entirely on scientific outcomes, to a focus that includes process as part of research outcomes.

To start, the authors provide a useful overview of PAR methods, tracing their disciplinary and scholarly origins. The list of the various schools of PAR and their theoretical sources and origins is both a helpful review for practitioners, and a fine introduction to those newly approaching this area of knowledge and practice. The second chapter is a useful reminder to practitioners of the unexpected and often invisible results of our research, in terms of power differentials and hierarchies, including timely warnings about the potential for unwitting coercion, and other factors in the research relationship. A critical discussion of the concept of empowerment forces the reader to be reflective and mindful with current research projects, since 'it is more productive to conceive the modalities of power and empowerment as entangled' (p. 23). Another key point is contributing to theory, as PAR has often been considered a-theoretical. In PAR the voice of the participants is included, giving rise to a range of challenges around confidentiality for academic ethics boards as they address this approach to research partnerships. Some papers provide guidance for very current challenges such as consistency of process versus uniformity of practice in ethical review and accountability, 
and researcher safety, a topic rarely addressed.

The second section of the book is focused on action, and includes a number of case studies with a range of participants such as New England fisheries, communities in Peruvian national parks, children in Zimbabwe and Kenya, and groups such as youth, disabled people and migrants. Each case study illustrates one or more PAR methods as applied in these unique environments. Tools illustrated include body mapping, peer strategies with youth, participatory art, diagramming, theatre and cartography, social auditing and photovoice, even participatory GIS.

The third section underlines that PAR is a process for learning for all participants, particularly the researchers. As an example, 'The different knowledge of parents, service users, service providers and researchers can be negotiated and boundaries collapsed' (p. 195). The authors also emphasise the importance of representing and communicating PAR; they advise researchers to check and doublecheck with all participants regarding the language and potential stereotyping in publications and other vehicles of dissemination as we link participatory research to action and thoughtful activism.

The papers in the book are uneven in terms of approach and depth, but all contribute to an understanding of the theory and practice of PAR. This is a useful textbook, as well as a tool for reviewing and expanding the approach and practice of experienced researchers. 